\title{
DEFICIENCY SETS AND BOUNDED INFORMATION REDUCIBILITIES
}

\author{
BY \\ LEONARD P. SASSO, JR. $\left({ }^{1}\right)$
}

\begin{abstract}
For recursively enumerable sets $A$ and $H$ of natural numbers $H$ is a deficiency set of $A$ if there is a one-one, recursive function $f$ with $A=\operatorname{Rng}(f)$ and $H=\{i:(\exists j)[i<j \& f(j)<f(i)]\}$. The relation between recursively enumerable sets and their deficiency sets under bounded information reducibilities (i.e. weak truth table, truth table, bounded truth table, many-one, and one-one reducibility) is investigated.
\end{abstract}

If $a_{0}, a_{1}, \cdots$ is a $1-1$, recursive enumeration of an r.e. set $A$ then $H_{a}=$ $\left\{i:(\exists j)\left[i<j \& a_{j}<a_{i}\right]\right\}$ is called a deficiency set of $A$. Deficiency sets were introduced by Dekker [2] who showed that $H_{a}$ must be hypersimple or recursive and that $A$ and $H_{a}$ are Turing equivalent. We are concerned with the relationship between r.e. sets and their deficiency sets under stronger, "bounded information" reducibilities introduced in Post [11] and Friedberg and Rogers [4]. We show that r.e. sets can have weak truth table incomparable deficiency sets or may be many-one equivalent to each of their deficiency sets. We show that weak truth table incomparable r.e. sets can share deficiency sets while oneone equivalent r.e. sets might not. We then improve these results using degree restricting techniques introduced in Soare [15], Yates [17], and below in $\S 1$. Drawing on work of Ladner [7] $\left({ }^{2}\right)$ we show, in most cases, that our degree restrictions are best possible. We also improve the results making use of Robinson's [13] dichotomy of the r.e. sets into high and low sets and of Rice's [12] notion of enumerability in the same order.

$\S 0$ is devoted to preliminaries including a brief survey of elementary results concerning deficiency sets, grades of simplicity, and high and low sets. In $\S 1$ we introduce the methods used in many of the proofs to follow and we consider reductions between sets and their deficiency sets and deficiency subsets. In $\S 2$ we

Received by the editors November 27, 1972 and, in revised form, September 24, 1973. AMS (MOS) subject classifications (1970). Primary 02F25, 02F30.

(1) I am indebted to C. G. Jockusch, Jr. for suggesting many of the questions considered in this paper and I am grateful to C. G. Jockusch, Jr., R. E. Ladner, and R. W. Robinson for valuable correspondence concerning this research. I am further indebted to the referee for helpful suggestions leading to the present formulation of $\S 1$ and of most of the proofs. Manuscript preparation supported by AFOSR Grant 71-2118.

(2) In [7] Ladner shows that each nonrecursive, r.e. degree has a nonrecursive, r.e. predecessor in which all r.e. sets are w-equivalent. 
consider r.e. sets with incomparable deficiency sets; in $\$ 3$ we consider incomparable r.e. sets with shared deficiency sets, and in $\$ 4$ we consider "self-deficient" sets and r.e. sets with many-one equivalent deficiency sets.

0 . Preliminaries. $N$ denotes the set of natural numbers $\{0,1,2, \cdots\}$ and upper case Latin letters except for $D, H$, and $W$ will be used as variables ranging over subsets of $N$. Lower case Latin letters will range over the natural numbers as well as over natural number functions of arbitrary dimension, the distinction being left to context. Subscripts will be used freely.

We adopt the following special conventions regarding finite sets: $A[k]=$ $\{n: n \in A \& n<k\}$. $|A|$ denotes the cardinality of $A$. If $n=2^{k_{1}}+2^{k_{2}}+$ $\cdots+2^{k_{m}}$ where $k_{1}<k_{2}<\cdots<k_{m}$ then $D_{n}=\left\{k_{1}, k_{2}, \cdots, k_{m}\right\} . D_{0}=$ $\varnothing . n$ is called the canonical index of $D_{n}$. $U D_{n}=\bigcup\left\{D_{m}: m \in D_{n}\right\}$.

We assume a fixed, standard enumeration of the unary, partial recursive functions (prf's) and let $\varphi_{e}$ denote the $e$ th prf. We use $\varphi_{e}^{t}$ to denote the prf obtained from $\varphi_{e}$ by allowing at most $t$ steps in computations under some fixed, standard step-counting procedure. We assume that $\varphi_{e}^{t}(n)$ is divergent (dgt) when $t<n$ and that $\varphi_{e}^{t+1}(n)$ is convergent (cgt) when $\varphi_{e}^{t}(n)$ is. We use $W_{e}$ to denote $\operatorname{Dom}\left(\varphi_{e}\right)$, call $W_{e}$ the $e$ th r.e. set, and call $e$ an r.e. index of $W_{e}$. We use $W_{e}^{t}$ to denote $\operatorname{Dom}\left(\varphi_{e}^{t}\right)$ whence $W_{e}^{0} \subseteq W_{e}^{1} \subseteq \cdots, W_{e}=\bigcup_{t} W_{e}^{t}$, and $W_{e}^{t} \subseteq\{n: n \leqslant t\}$. We call a one-one recursive function $a$ an enumeration of its range and often write $a_{i}$ for $a(i)$. We use the recursive, one-one, pairing function $\langle\cdot, \cdot\rangle$ with projections $(\cdot)_{0}$ and $(\cdot)_{1}$ given by $\langle m, n\rangle=2^{m}(2 n+1)$ -1 and $(\langle m, n\rangle)_{0}=(\langle n, m\rangle)_{1}=m$ for all $m$ and $n$.

The notions of deficiency set and deficiency subset with which we are concerned derive from notions introduced in Dekker [2]. We define the deficiency set $H_{a}$ of an enumeration $a$ by $H_{a}=\left\{i:(\exists j)\left[i<j \& a_{j}<a_{i}\right]\right\}$ and we define the deficiency subset $H_{a}^{*}$ by $H_{a}^{*}=\left\{a_{i}: i \in H_{a}\right\}$. We use recursive approximations to $H_{a}$ and $H_{a}^{*}$ defined by

$$
H_{a}^{k}=\left\{i:(\exists j)\left[i<j<k \& a_{j}<a_{i}\right]\right\} \quad \text { and } \quad\left(H_{a}^{k}\right)^{*}=\left\{a_{i}: i \in H_{a}^{k}\right\} .
$$

(Note that, in general, $H_{a}^{k} \neq H_{a}[k]$ and $\left(H_{a}^{k}\right)^{*} \neq H_{a}^{*}[k]$.) $H_{a}\left(H_{a}^{*}\right)$ is called a deficiency set (subset) of Rng (a).

Dekker's purpose in introducing deficiency sets, showing as he did that $H_{a}$ and Rng $(a)$ have the same degree of unsolvability and that $H_{a}$ is hypersimple when Rng ( $a$ ) is not recursive, was to exhibit a "natural" hypersimple set in each r.e. degree $>\mathbf{0}$. Recall that Post [11] defines (hyper)hypersimple sets as those co-infinite, r.e. sets which contain at least one member of each disjoint (weak) array. (Here a disjoint (weak) array is an infinite set of pairwise disjoint finite sets such that the set of their canonical indices (a set containing exactly 
one r.e. index of each) is r.e.) Yates [16] has shown that deficiency sets cannot be hyperhypersimple by showing that hyperhypersimple sets cannot have retraceable complements while, as shown by Dekker and Myhill [3], deficiency sets always have retraceable complements. (A co-r.e. set $B=\left\{b_{0}<b_{1}<\cdots\right\}$ is retraceable if there is an rf $g$ such that $g\left(b_{0}\right)=b_{0}$ and $(\forall i)\left[g\left(b_{i+1}\right)=b_{i}\right]$.) Martin [8] and [9] defines two intermediate grades of simplicity, dense-simple and supersimple (also called finite strongly hypersimple), only the first of which is possible for deficiency sets. His definition of supersimplicity is parallel to Post's definition of hypersimplicity but with respect to arrays enumerable by characteristic indices rather than canonical indices. Martin observes that Yates' proof that hyperhypersimple sets cannot have retraceable complements is easily modified to apply to supersimple sets. Martin's definition of dense-simplicity is parallel to an alternate definition of hypersimplicity due to Rice [12]. Rice shows that a co-infinite, r.e. set is hypersimple just if its complement is not dominated by any rf. (A function $g$ dominates a function $f$ if $\{n: g(n) \leqslant f(n)\}$ is finite and a set $A$ dominates a set $B$ if the principal function of $A$, the function listing $A$ in increasing order, dominates principal function of $B$.) Martin [9] calls co-infinite, r.e. set dense-simple if its complement dominates every recursive set. He shows in [8] that hyperhypersimple sets are dense-simple. (Dense-simple sets are clearly hypersimple by Rice's definition.) Robinson [13] shows that deficiency sets can be dense-simple by showing that his high sets have only dense-simple deficiency sets. He also shows that his low sets can have densesimple deficiency sets. It follows that "thickness" of deficiency sets is not a reliable measure of how fast initial segments of an r.e. set can be captured by an enumeration.

Robinson's [13] notion of high and low for r.e. sets is, as he shows, a reliable measure of the speed with which initial segments may be captured, initial segments of low sets being more quickly capturable than initial segments of high sets. With any recursive function $f$ such that, for all $m<n, D_{f(m)} \subseteq D_{f(n)}$ he associates a computation function $c$ for the r.e. set $A=\bigcup_{m} D_{f(m)}$ defined by $c(k)=$ the least $m$ with $A[k]=D_{f(m)}[k]$ (i.e., $c(k)$ is the least "stage" at which all elements of $A[k]$ have appeared). Clearly $A$ is recursive just if $c$ is dominated by some recursive function. Robinson calls $A$ high if $c$ dominates every recursive function and calls $A$ low otherwise. He shows that the notions are well defined by showing that all computation functions for $A$ dominate all recursive functions if any does. He also shows that $A \leqslant_{\mathrm{w}} B$ cannot hold if $A$ is high while $B$ is low and that high sets appear in just those r.e. degrees with jump $\mathbf{0}^{\prime \prime}$. In proving the latter he makes use of Martin's proof [9] that densesimple sets are contained in just such degrees. Robinson [15] also shows that low sets appear in all r.e. degrees. This follows (although he uses a direct construc- 
tion) from the observation that co-retraceable, r.e. sets (deficiency sets in particular) are low. To see this let $f$ and $c$ be as above and suppose $\bar{A}$ is retraced by a recursive function $g$. Then let $h$ and $k$ be recursive functions such that $n+1<\left|N-\left(D_{f(n)}\right)[k(n)]\right|$ for each $n$ while $g$ retraces $N-\left(D_{f(n)}\right)[k(n)]$ for each $n$. It follows that $D_{f(n)}[n]=A(n)$ for each $n \in \bar{A}$ whence $A$ is low.

As stated in the introduction we are concerned with the relation between r.e. sets and their deficiency sets under "bounded information" reducibilities. By bounded information reducibilities we mean one-one, many-one, bounded truth table, and truth table reducibility as introduced by Post in [11] and weak truth table reducibility as defined by Friedberg and Rogers in [4]. We refer to these reducibilities as $1, \mathrm{~m}, \mathrm{btt}, \mathrm{tt}$, and w-reducibility respectively and we denote by $\leqslant$ with the appropriate subscript the partial ordering of the r.e. sets induced by each reducibility.

We refer the reader to Friedberg and Rogers [4] for a detailed account of these reducibilities as they apply to r.e. sets. Intuitively we think of a reduction of an r.e. set $A$ to an r.e. set $B$ as affected by an $\mathrm{rf} g$ which gives correct information about $\bar{A}$ based on knowledge of $\bar{B}$. As a 1-or m-reduction we say that $g(n)$ succeeds for $\bar{B}$ if $g(n) \in \bar{B}$. As a btt- or tt-reduction we say that $g(n)$ succeeds for $\bar{B}$ if $(\exists k)\left[k \in D_{\underline{g(n)}} \& D_{k}=\bar{B} \cap \bigcup D_{g(n)}\right]$. As a w-reduction we say that $g(n)$ succeeds for $\bar{B}$ if $(\exists k)\left[k \in D_{(g(n))_{0}} \cap W_{(g(n))_{1}}\right.$ \& $\left.D_{k}=\bar{B} \cap \bigcup D_{(g(n))_{0}}\right]$. Then $A$ is $m$, $t t$-, or $w$-reducible to $B$ if there is an rf $g$ such that $(\forall n)[n \in \bar{A} \leftrightarrow g(n)$ succeeds for $\bar{B}]$ where "succeeds" is interpreted appropriately. $A$ is 1-reducible to $B$ if $A$ is m-reducible to $B$ by a one-one rf. $A$ is btt-reducible to $B$ if $A$ is tt-reducible to $B$ by an $\mathrm{rf} g$ for which there is a uniform bound $b$ such that $\left|\bigcup D_{g(n)}\right|<b$ for all $n$.

Notice that in all but the w-reducibility case we can determine from knowledge of $\bar{B}$ whether or not $g(n)$ succeeds for $\bar{B}$. In the w-reducibility case $g(n)$ may "fail" for $\bar{B}$ relative to $W_{(g(n))_{1}}^{t}$ while succeeding relative to $W_{(g(n))_{1}}$. Hence in stage-by-stage constructions to follow, when at a stage $t$ we speak of failure in the sense of w-reducibility we intend the obvious restriction.

It is easy to verify for any enumeration $a$ of any r.e. set $A$ that $H_{a} \leqslant_{\mathrm{tt}} A$ and that $H_{a} \leqslant_{\mathrm{m}} H_{a}^{*} \leqslant_{\mathrm{w}} A$. We will illustrate the methods introduced in $\S 1$ by arguments designed to show that $H_{a}$ is not generally btt-reducible to $A$ and that $H_{a}^{*}$ is not generally tt-reducible to $A$. On the other hand, it follows from work of Jockusch in [5] that $H_{a} \leqslant_{\mathrm{m}} A$ when $A$ is co-retraceable. This result will be useful in $\S 4$, Theorem 9 where we construct an r.e. set $A$ m-equivalent to each of its deficiency sets. In the other direction, Dekker [2] shows that any r.e. set is Turing reducible to each of its deficiency sets. Robinson's proof in [13] that high sets are never w-reducible to low sets together with our observation that 
deficiency sets are always low show that r.e. sets are not generally w-reducible to their deficiency sets. Also Post's [11] proof that hypersimple sets cannot be $\mathrm{tt}$-complete is easily modified to show that hypersimple sets cannot be w-complete.

1. Reductions between sets and their deficiency sets. In $\S 4$ of [14] Sacks formalizes the combinatorial aspect of a class of finite injury priority constructions. In Sacks' formalism a requirment is an effectively open set in the Cantor topology on $2^{N}$ and a set $A \subseteq N$ meets a requirement by lying in the corresponding open set. In Sacks' constructions each condition to be satisfied by the set $A$ being enumerated is associated with a single requirement which, if met, ensures that the condition is satisfied. The enumeration of requirements together with the enumeration of $A$ is designed to ensure that nonvacuous conditions have requirements which are met.

With the exception of Theorems 8 and 9 our constructions could employ Sacks' combinatorial formalism although our conditions would need to be associated with several requirements each of which would have to be met to ensure a nonvacuous condition. In the bounded truth table and truth table constructions two requirements are needed per condition; in the one-one and weak truth table construction three requirements are needed; and in the construction for Theorem 10 nola priori bound exists on the number of requirements needed by a given condition. In this last case a variable assignment of requirements to conditions is needed but the assignment settles down because each condition needs only finitely many requirements. We will not employ Sacks' formalism in our constructions because intuition is better served by another approach.

In [15] Soare gives sufficient conditions for completeness of a set enumerated in a Sacks' construction. We apply his completeness criterion in Theorems $4,6,7$ and 10 to show that complete sets with the indicated properties exist. In [15] Soare also shows how to apply the permitting method introduced in Yates [17] to any Sacks' construction to push down the degree of the resulting set. In applying this method when conditions are associated with several requirements care must be taken first to ensure that each condition requires only finitely many requirements and second to ensure that requirements of a nonvacuous condition are met in the correct order. Both considerations can be met in the one-one, bounded truth table, truth table, and weak truth table constructions to follow but the first consideration cannot be met in the construction of Theorem 10. In the bounded truth table and truth table constructions, partly because each condition needs only two requirements the first of which is highly flexible, a combination of permitting and coding which we call trapping may be applied to give the resulting set any desired r.e. degree $>0$.

In our constructions each condition will have a unique natural number 
index and lower numbered conditions will be given higher priority. The constructions are conceived in stages with each stage devoted to a single condition. (We will systematically confuse stages with stage numbers.) At any stage some earlier stages may be designated as invalid. A stage is valid until (if ever) designated invalid and is invalid thereafter. Some sequences of currently valid stages devoted to the same condition will be called attacks on that condition and the first stage in an attack will be called an initial attack on that condition. Circumstances will be given under which a condition needs an initial attack and under which an existing attack needs attention. An attack which needs attention will continue to need attention until (if ever) it receives attention or is invalidated. A condition is said to need attention if either it needs an initial attack or it has an attack needing attention. In the latter case the condition is said to need attention through the attack. In constructions which employ permitting or trapping we may be prohibited from giving attention through a particular attack but modulo such prohibitions we devote each construction stage to the highest priority condition in need. An attack is said to win if it never needs attention and if none of its stages ever becomes invalid. Proving that a construction works entails showing that each condition has a winning attack.

A basic construction is one in which neither permitting nor trapping is employed. At a basic construction stage we automatically designate as invalid all earlier stages devoted to lower priority conditions. In a basic construction a condition needs an initial attack if and only if it has no current attacks (hence a condition has at most one attack at a time). We devote a basic construction stage to the highest priority condition needing attention. Hence proving that a basic construction works entails showing that any attack whose stages are never invalidated has a winning extension (perhaps itself).

In all constructions a top-marking function $m(t)$, nondecreasing in stages $t$, is used to prevent initial attacks from interfering with existing attacks. $(m(t)$ may be viewed as incorporating all "restraints" needed to protect existing attacks.) An initial attack at stage $t$ entails putting number(s) larger than $m(t)$ into the set $A$ being constructed and/or designating other numbers larger than $m(t)$ as followers of this attack (perhaps to be put into $A$ at stages extending the attack). $m(t+1)$ is then taken to be the maximum of all followers, all numbers put into $A$, and all "restraints" necessary to preserve this attack. Extending (i.e., giving attention to) an attack at stage $t$ entails putting some followers of that attack into $A$ and defining $m(t+1)$ to exceed all "restraints" now needed to preserve the attack.

In order to show that a set $A$ enumerated in a basic construction is complete it suffices (cf. Soare [15, Theorem 2]) to find for each $e$, a condition whose winning attack contains a single stage if and only if $e \notin W_{e}$. It follows 
that $e \notin W_{e}$ if and only if there is a stage $t$ devoted to an initial attack on this condition and such that no number below $m(t+1)$ enters $A$ after stage $t$. (Recall that in basic constructions attention goes to the highest priority condition in need.)

In applying permitting or trapping we suppose we are given an enumeration $b$ of a nonrecursive, r.e. set $B$. We let $B^{t}=\left\{b_{i}: i<t\right\}$ and we let $A^{t}$ denote the set being constructed as constituted prior to stage $t$. In permitting we ensure that $A^{t+1}\left[b_{t}\right]=A^{t}\left[b_{t}\right]$ for each $t$. It follows for each $n$ that $A[n]=A^{t}[n]$ if $B[n]=B^{t}[n]$ whence $A \leqslant{ }_{\mathrm{w}} B$ and a fortiori $\mathbf{A} \leqslant \mathbf{B}$.

In applying trapping we use a partial coding function $c(n, t)$ to code $B$ in $A$ and to restrict changes in $A$ modulo $b$. At each stage $t, c(n, t)$ will be defined on a finite initial segment with $c(0, t)=0$ and $c(n, t)<c(n+1, t)$ if $c(n+1, t)$ is defined. If $c\left(1+b_{t}, t\right)$ is defined it will enter $A$ at stage $t$, $c(n, t+1)$ will be undefined for all $n>1+b_{t}$, and $c(n, t)=c(n, t+1)$ for all $n \leqslant 1+b_{t}$. If $c\left(1+b_{t}, t\right)$ is not defined then $c(n, t)=c(n, t+1)$ for all $n$ with $c(n, t)$ defined and $c(n, t+1)$ may (or may not) be defined for the least $n$ with $c(n, t)$ undefined. (All other $c(n, t+1)$ will be undefined.) There will be infinitely many stages $t$ with $c\left(1+b_{t}, t\right)$ undefined at which a new value $c(n, t+1)$ will be defined. It follows (since $b$ is $1-1)$ that for each $n$ there is a single value taken by $c(n, t)$ at all sufficiently late stages $t$. Hence for each $n$ there is a stage $t$ with $c(n+1, t)$ defined and with $A[1+c(n+1, t)]=A^{t}[1+c(n+1, t)]$. It follows that $B[n+1]=B^{t}[n+1]$ whence, since the construction is recursive, $B \leqslant_{T} A$. Also for each $m$ there is a stage $t$ and an $n$ such that $c(n+1, t)$ is defined, $m \leqslant c(n+1, t)$, and $B[n+1]=B^{t}[n+1]$. It follows that $A[1+c(n+1, t)]=A^{t}[1+c(n+1, t)]$ (in particular $m \in A$ if and only if $m \in A^{t}$ ) whence, since the construction is recursive, $A \leqslant_{T} B$. (We will in fact have that $c(n, t) \leqslant c\left(n, t^{\prime}\right)$ when both are defined and $t \leqslant t^{\prime}$. From this it follows that $A \leqslant{ }_{\mathrm{w}} B$.)

We begin each proof with a basic construction to illustrate the combinatorial devices needed to satisfy the conditions. We then indicate how to apply Soare's [15] completeness criterion and permitting or trapping where possible. Theorem 1 is meant to serve as a paradigm basic and trapping construction. Theorem 4 is meant to serve as a paradigm completeness and permitting construction.

THEOREM 1. Every r.e. degree $>0$ contains an r.e. set $A$ with a deficiency set $H$ not btt-reducible to $A$.

Proof. We define an enumeration $a$ of an r.e. set $A$ such that $H_{a}$ $\star_{b t t} A$. We define $a_{t}$ at stage $t$ and let $A^{t}=\left\{a_{i}: i<t\right\}$. We take as our $\langle n, b\rangle$ th condition that $\varphi_{n}$ not be a tt-reduction of $H_{a}$ to $A$ with bound $b$. If stage $t$ is an initial attack on this condition we let $m(t+1)=a_{t}=m(t)+$ 
$b+1$. We designate all numbers between $m(t)$ and $a_{t}$ as followers of this attack. If at stage $t^{\prime}>t$ we find that $\varphi_{n}(t)$ succeeds for $N-\left(A^{t^{\prime}}\right)$ while $\left|\bigcup D_{\varphi_{n}(t)}\right|<b$ then one of these $b$ followers may be used to put $t$ in $H_{a}$ without changing the success of $\varphi_{n}(t)$ for $\bar{A}$. We say that such an attack initiated at stage $t$ needs attention at stage $t^{\prime}>t$ if it has not received attention at any intermediate stage (i.e., at any stage $t^{\prime \prime}$ with $t<t^{\prime \prime}<t^{\prime}$ ) and if $\varphi_{n}^{t^{\prime}}(t)$ converges while $\left|\bigcup D_{\varphi_{n}(t)}\right|<b$. Such attention is given in one of two ways. If $\varphi_{n}(t)$ succeeds for $N-\left(A^{t^{\prime}}\right)$ then we let $a_{t^{\prime}}$ be the largest follower of this attack not in $\bigcup D_{\varphi_{n}(t)}$. If $\varphi_{n}(t)$ fails for $N-\left(A^{t^{\prime}}\right)$ we let $a_{t^{\prime}}=1+$ $\operatorname{Max}\left(\left\{m\left(t^{\prime}\right)\right\} \cup \cup D_{\varphi_{n}(t)}\right)$. In both cases we let $m\left(t^{\prime}+1\right)=1+$ $\operatorname{Max}\left(\left\{m\left(t^{\prime}\right)\right\} \cup \bigcup D_{\varphi_{n}(t)}\right)$.

Under these circumstances of needing attention and the basic construction rules for giving attention to the highest priority condition in need each condition will have a winning attack. If the winning attack for the $\langle n, b\rangle$ th condition contains a single stage $t$ then $\varphi_{n}(t)$ diverges or $\left|\bigcup D_{\varphi_{n}(t)}\right| \geqslant b$. If this attack has two stages $t<t^{\prime}$ then either $\varphi_{n}(t)$ succeeds for $N-\left(A^{t^{\prime}}\right)$ and $t \in H_{a}$ (because $\left.a_{t^{\prime}}<a_{t}\right)$ while $\varphi_{n}(t)$ succeeds for $\bar{A}$ or $\varphi_{n}(t)$ fails for $N-\left(A^{t^{\prime}}\right)$ and $t \notin H_{a}$ (because $a_{t}<m\left(t^{\prime}\right)<a_{t^{\prime}}$ ) while $\varphi_{n}(t)$ fails for $\bar{A}$. (In both cases success or failure for $\bar{A}$ is ensured by the definition of $m\left(t^{\prime}+1\right)$ and the continued validity of stage $t^{\prime}$.) In any case $\varphi_{n}$ is not a tt-reduction of $H_{a}$ to $A$ with bound $b$.

In applying trapping to this construction we may need to define more than one value of $a$ at a given stage. We use a marking function $j(t)$ defined as the least $i$ with $a_{i}$ undefined prior to stage $t$. We let $A^{t}=\left\{a_{i}: i<j(t)\right\}$. (If stage $t$ is devoted to an initial attack then only one value, $a_{j(t)}$, will be defined whence any extension of this attack will be used to put $j(t)$ in or out of $H_{a}$.)

For any two attacks the one initiated at the earlier stage is said to be earlier than the other. It is important to observe that by use of the top-marking function $m(t)$ we ensure that all followers of an earlier attack precede all followers of a later attack. We wish to keep all followers of a given attack either above all defined coding markers $c(n, t)$ or between a consecutive pair $c(n, t)$ and $c(n+1, t)$ of coding markers. If all of an attack's followers are between $c(n, t)$ and $c(n+1, t)$ we say the attack is in the $n$th trap. It follows from preceding observation that earlier attacks will be in equal or lower (numbered) traps. We will ensure when defining $c(n+1, t+1)$ that the $n$th trap contains an attack for each condition of priority higher (lower index) than $n+1$.

It is necessary to ensure in trapping constructions that an attack needing attention continues to need attention until attended to or invalidated. It is also necessary to ensure that if any attack on a condition needs attention then 
all earlier attacks on that condition (which are still valid) also need attention. We do so by stipulation.

A condition needs an initial attack at stage $t$ if each of its current attacks is in a trap. An attack on the $\langle n, b\rangle$ th condition initiated at stage $t$ needs attention at stage $t^{\prime}$ if $\varphi_{n}^{t^{\prime}}(j(t))$ is defined, $\left|\bigcup D_{\varphi_{n}(j(t))}\right|<b$, it has not received attention at any intermediate stage, and all current, earlier attacks on the $\langle n, b\rangle$ th condition need attention.

Let an enumeration $b$ of a nonrecursive, r.e. set $B$ be given.

Case 1: $c\left(1+b_{t}, t\right)$ is defined. Let $a_{j(t)}=c\left(1+b_{t}, t\right)$ and consider invalid all stages in all attacks not in the $b_{t}$ th or lower traps. If some attack in the $b_{t}$ th trap needs attention let the $\langle n, b\rangle$ th be the highest priority condition with such an attack. There will be only one such attack for the $\langle n, b\rangle$ th condition and this will be the earliest attack in the $b_{t}$ th trap needing attention. If this attack was initiated at stage $t^{\prime}$ and if $\varphi_{n}\left(j\left(t^{\prime}\right)\right)$ succeeds for $N-$ $\left(A^{t} \cup\left\{a_{j(t)}\right\}\right)$ then let $a_{j(t)+1}$ be the largest follower of this attack not in

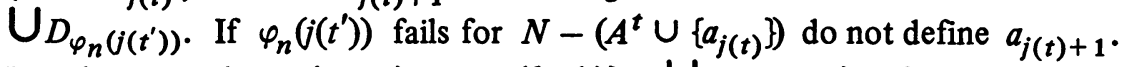
In either event let $m(t+1)=\operatorname{Max}\left(\{m(t)\} \cup \cup D_{\varphi_{n}\left(j\left(t^{\prime}\right)\right)}\right)$. If no attack in the $b_{t}$ th trap needs attention do not define $a_{j(t)+1}$ and let $m(t+1)=m(t)$.

Case 2: $c\left(1+b_{t}, t\right)$ is not defined. Let the $\langle n, b)$ th be the highest priority condition needing an initial attack. Let $m(t+1)=a_{j(t)}=m(t)+b+1$ and designate all numbers between $m(t)$ and $a_{j(t)}$ as followers of this attack. If $k$ is minimal with $c(k+1, t)$ not defined and $k \leqslant\langle n, b\rangle$ then define $c(k+1, t+1)=m(t+1)+1$. Otherwise leave $c(k+1, t+1)$ undefined.

By arguments in the basic construction any condition with a winning attack is satisfied. This is so because success or failure in case of a two stage winning attack will be protected by the top-marking function against all attacks in higher traps than the trap of the winning attack and attention to attacks in lower traps would have invalidated this winning attack. We are thus left with showing that each condition has a winning attack.

If some condition has no winning attack let the $\langle n, b\rangle$ th be the highest priority such condition. We know that all but the first $\langle n, b\rangle$ "permanent traps" will contain attacks on the $\langle n, b\rangle$ th condition which are never invalidated and which need attention at all sufficiently late stages. Hence modulo $B[\langle n, b\rangle]$ and winning attacks on higher priority conditions we can conclude that $B[k]=$ $B^{t}[k]$ for $k \geqslant\langle n, b\rangle$ if $c(k+1, t)$ is defined and the $k$ th trap has an attack on the $\langle n, b\rangle$ th condition which needs attention. This is so because all traps between the $\langle n, b\rangle$ th and the $k$ th will then contain attacks on the $\langle n, b\rangle$ th condition which need attention and this need will be persistent. Then the smallest number $k^{\prime}$ with $\langle n, b\rangle \leqslant k^{\prime} \leqslant k$ to enter $B$ after stage $t$ would lead to a winning attack on the $\langle n, b\rangle$ th condition. But given that $B[\langle n, b\rangle]=$ 
$B^{t} 0[\langle n, b\rangle]$ and that no condition of priority higher than the $\langle n, b\rangle$ th has an attack extended after the stage $t_{0}$ such a stage $t>t_{0}$ can be found for each $k$. Since this is contrary to the hypothesis that $B$ is not recursive all conditions must have winning attacks.

TheOREM 2. Every r.e. degree $>0$ contains an r.e. set $A$ with a deficiency subset $H^{*}$ not tt-reducible to $A$.

Proof. We define an enumeration $a$ of an r.e. set $A$ such that $H_{a}^{*} \$_{\mathrm{tt}}$ $A$. Since we will not add numbers to $A$ at some stages we let $j(t)$ be the least $i$ with $a_{i}$ undefined prior to stage $t$ and let $A^{t}=\left\{a_{i}: i<j(t)\right\}$. We take as our $e$ th condition that $\varphi_{e}$ not be a tt-reduction of $H_{a}^{*}$ to $A$. If stage $t$ is an initial attack on the $e$ th condition we let $m(t+1)=m(t)+2$ and designate $m(t)+1$ and $m(t)+2$ as followers of this attack. In extending this attack we will put both followers into $A$ and depending on case we may or may put the larger follower, $m(t)+2$, into $H_{a}^{*}$. We say that such an attack initiated at stage $t$ needs attention at stage $t^{\prime}>t$ if it has not received attention at any intermediate stage and if $\varphi_{e}^{t^{\prime}}(m(t)+2)$ converges. In giving such attention we distinguish between cases where $\varphi_{e}(m(t)+2)$ succeeds and fails for $A^{t^{\prime}} \cup\{m(t)+1, m(t)+2\}$. In case of success we let $a_{j\left(t^{\prime}\right)}=m(t)+1$ and $a_{j\left(t^{\prime}\right)+1}=m(t)+2$ and in case of failure we let $a_{j\left(t^{\prime}\right)}=m(t)+2$ and $a_{j\left(t^{\prime}\right)+1}$ $=m(t)+1$. In either case we let $m\left(t^{\prime}+1\right)=\operatorname{Max}\left(\{m(t)\} \cup \bigcup D_{\varphi_{e}}(m(t)+2)\right)$.

Now in a single stage winning attack on the $e$ th condition, say at stage $t$, we have that $\varphi_{e}(m(t)+2)$ diverges. In a two stage winning attack, say at stages $t<t^{\prime}$, we have either that $m(t)+2 \notin H_{a}^{*}$ while $\varphi_{e}(m(t)+2)$ succeeds for $\bar{A}$ or $m(t)+2 \in H_{a}^{*}$ while $\varphi_{e}(m(t)+2)$ fails for $\bar{A}$. In any case $\varphi_{e}$ is not a tt-reduction of $H_{a}^{*}$ to $A$.

Applying trapping in this construction is completely analogous to its application in the proof of Theorem 1. The need for attention persists automatically, we stipulate that the need for attention progresses through earlier to later attacks, and we define new coding markers when enough initial attacks have been made. In distinguishing the success and failure cases at stage $t^{\prime}$ we again include $c\left(1+b_{t^{\prime}}, t^{\prime}\right)$ in $A$.

Recall from the preliminaries that for any r.e. set $A$ with enumeration $a$, $H_{a} \leqslant_{\mathrm{tt}} A$ and $H_{a}^{*} \leqslant_{\mathrm{w}} A$. Theorems 1 and 2 show that, relative to any r.e. degree $>0$, this is the best that can be counted on.

2. Incomparable deficiency sets. We will show in this section that an r.e. set may have w-incomparable deficiency sets and deficiency subsets, that $\mathbf{0}^{\prime}$ contains such a set, that every r.e. degree $>0$ has a predecessor $>0$ containing such a set, and that in the case of tt-incomparability every r.e. degree $>0$ con- 
tains such a set. We know from previously cited work of Ladner that these degree restrictions for w-reducibility are best possible. Theorem 4 of this section serves as a paradigm permitting construction and application of Soare's completeness criterion.

Recall from the preliminaries that $H_{a} \leqslant_{\mathrm{m}} H_{a}^{*}$ for any enumeration $a$. Following a suggestion of $\mathrm{R}$. W. Robinson we use this to obtain the deficiency set and deficiency subset results simultaneously. Specifically we obtain enumerations $a$ and $a^{\prime}$ of an r.e. set $A$ such that $H_{a} \$_{\mathrm{tt}} H_{a^{\prime}}^{*}$ and $H_{a^{\prime}} \$_{\mathrm{tt}} H_{a}^{*}$ and similarly for w-reducibility. It follows that $H_{a}$ and $H_{a^{\prime}}$ are incomparable as are $H_{a}^{*}$ and $H_{a^{\prime}}^{*}$ since if, for example, $H_{a} \leqslant_{\mathrm{tt}} H_{a^{\prime}}$ then $H_{a} \leqslant_{\mathrm{tt}} H_{a^{\prime}} \leqslant_{\mathrm{m}}$ $H_{a^{\prime}}^{*}$ whence $H_{a} \leqslant_{\mathrm{tt}} H_{a^{\prime}}^{*}$.

THEOREM 3. Every r.e. degree $>0$ contains an r.e. set $A$ with enumer-

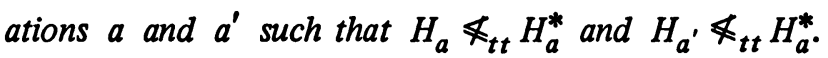

Proof. We find values of $a$ and $a^{\prime}$ simultaneously so that $\left\{a_{i}: i<j(t)\right\}$ $=\left\{a_{i}^{\prime}: i<j(t)\right\}$ at each stage $t$. (Here as before $j(t)$ is the least $i$ with $a_{i}$ undefined prior to stage $t$ and $A^{t}=\left\{a_{i}: i<j(t)\right\}$.) We take as our 2eth condition that $\varphi_{e}$ not be a tt-reduction of $H_{a}$ to $H_{a^{\prime}}^{*}$ and as our $(2 e+1)$ st condition $\varphi_{e}$ not be a tt-reduction of $H_{a^{\prime}}$ to $H_{a}^{*}$. If stage $t$ is an initial attack on the $(2 e+k)$ th condition (where $k \leqslant 1$ ) we put $4 m(t)+1$ and $4 m(t)+3$ into $A$ by defining

$a_{j(t)+k}=4 m(t)+1=a_{j(t)+(1-k)}^{\prime} \quad$ and $\quad a_{j(t)+(1-k)}=4 m(t)+3=a_{j(t)+k}^{\prime}$.

We designate $4 m(t)+2$ and $4 m(t)+4$ as followers of this attack and define $m(t+1)=m(t)+4$. If $k=0$ we have put $4 m(t)+3=a_{j(t)}^{\prime}$ into $H_{a^{\prime}}^{*}$ while keeping $j(t)+1$ out of $H_{a}$. At a later stage we may put either follower $4 m(t)+2$ or $4 m(t)+4$ into $A$ with the same effect on $H_{a^{\prime}}^{*}$ but with the effect of putting $j(t)+1$ into $H_{a}$ only in the first case. (Similar remarks apply if $k=1$.) We say that this attack needs attention at stage $t^{\prime}>t$ if it has not received attention at any intermediate stage and if $\varphi_{e}^{t^{\prime}}(j(t)+1)$ converges. Again we distinguish two cases. If $\varphi_{e}(j(t)+1)$ succeeds for $N-$ $\left(\left(H_{a^{\prime}}^{j\left(t^{\prime}\right)}\right)^{*} \cup\left\{a_{i}^{\prime}: j(t)+1<i<j\left(t^{\prime}\right)\right\}\right)$ we put $4 m(t)+2$ in $A$ by defining $a_{j\left(t^{\prime}\right)}=4 m(t)+2=a_{j\left(t^{\prime}\right)}^{\prime}$. Otherwise (i.e. in case of failure) we put $4 m(t)+4$ into $A$ by defining $a_{j\left(t^{\prime}\right)}=4 m(t)+4=a_{j\left(t^{\prime}\right)}^{\prime}$. In either case we let $m\left(t^{\prime}\right)=$ $\operatorname{Max}\left(\left\{m\left(t^{\prime}\right)\right\} \cup \cup D_{\varphi_{e}(j(t)+1)}\right)$.

Now in case of a single stage winning attack we have a divergence of $\varphi_{e}$ as in Theorem 2. In case of a two-stage winning attack, say on the $2 e$ th condition, containing stages $t<t^{\prime}$ we will have put $j(t)+1$ into $H_{a}$ only in the case of success while in either case, we will have ensured that $H_{a}^{*}\left[m\left(t^{\prime}+1\right)\right]=$ $\left(H_{a^{\prime}}^{j}\left(t^{\prime}\right) * \cup\left\{a_{i}^{\prime}: j(t)+1<i<j\left(t^{\prime}\right)\right\}\right.$ since stage $t^{\prime}$ is valid at all later stages. 
It follows that $\varphi_{e}$ is not a tt-reduction of $H_{a}$ to $H_{a}^{*}$. Similar arguments apply to odd numbered conditions.

The application of trapping to this construction is again analogous to its application in Theorem 1.

TheOREM 4. Below every r.e. degree $>\mathbf{0}$ there is an r.e. degree $>\mathbf{0}$ containing $a$ set $A$ with enumerations $a$ and $a^{\prime}$ such that $H_{a} \sharp_{w} H_{a^{\prime}}^{*}$ and $H_{a^{\prime}} \&_{w} H_{a}^{*}$. Furthermore, $0^{\prime}$ contains such a set.

PROof. We proceed as in Theorem 3 except that a two stage attack may need attention when success and failure are taken in the sense of w-reducibility. Specifically, if at stage $t^{\prime}$ of a two stage attack, say on the $2 e$ th condition, containing stages $t<t^{\prime}$ we find that $\varphi_{e}(j(t)+1)$ fails for $N-\left(\left(H_{a}^{j\left(t^{\prime}\right)}\right)^{*} U\right.$ $\left.\left\{a_{i}^{\prime}: j(t)+1<i<j\left(t^{\prime}\right)\right\}\right)$ and proceed as in Theorem 3 we may find at a stage $t^{\prime \prime}>t^{\prime}$ that, owing to a change in $W_{\left(\varphi_{e}(j(t)+1)\right)_{1}}, \varphi_{e}(j(t)+1)$ now succeeds for $N-\left(\left(H_{a}^{j\left(t^{\prime}\right)}\right)^{*} \cup\left\{a_{i}^{\prime}: j(t)+1<i<j\left(t^{\prime}\right)\right\}\right)$. Fortunately, we still have $4 m(t)+$ 2 which may be put into $A$ thus putting $j(t)+1$ into $H_{a}$ while leaving $H_{a}^{*}\left[m\left(t^{\prime}+1\right)\right]$ unchanged. The above mentioned success is now protected by $m\left(t^{\prime}+1\right)$ as long as stage $t^{\prime}$ remains valid.

We say that a two stage attack on the $2 e$ th condition which contains stages $t<t^{\prime}$ needs attention at stage $t^{\prime \prime}>t^{\prime}$ if $4 m(t)+2 \notin A^{t^{\prime \prime}}$ while $\varphi_{e}(j(t)+1)$ succeeds for $N-\left(\left(H_{a}^{j\left(t^{\prime}\right)}\right)^{*} \cup\left\{a_{i}^{\prime}: j(t)+1<i<j\left(t^{\prime}\right)\right\}\right)$ at stage $t$ ". (Note that the absence of $4 m(t)+2$ from $A$ indicates the "failure case" at stage $t^{\prime}$. Observe also that by the definition of $m\left(t^{\prime}+1\right)$ this new need for attention will persist until, if ever, stage $t^{\prime}$ becomes invalid.) If this attack receives attention at stage $t^{\prime \prime}$ we define $a_{j\left(t^{\prime \prime}\right)}=4_{m(t)+2}=a_{j\left(t^{\prime \prime}\right)}^{\prime}$ and $m\left(t^{\prime \prime}+1\right)=m\left(t^{\prime \prime}\right)$. The definitions and actions for odd numbered conditions are similar. Single and two stage winning attacks ensure their conditions as in Theorem 3. Three stage winning attacks ensure their condition by arguments in the preceding paragraph.

To see that $A$ is complete observe that for each $k$ we may find an index $e_{k}$ such that for each $j, \varphi_{e_{k}}(j)$ converges if and only if $k \in W_{k}$ and in the case of convergence

$$
D_{\left(\varphi_{e_{k}}(j)\right)_{0}}=W_{\left(\varphi_{e_{k}}(j)\right)_{1}}=\{1\}
$$

Since $D_{1}=\{\varnothing\}$ we have for each $j$ and $k$ that $\varphi_{e_{k}}(j)$ succeeds for $\varnothing$ (and hence for $\left.H_{a}^{*}\right)$ if and only if $k \in W_{k}$. Then by remarks in $\S 1 A$ is complete.

To apply permitting to this construction suppose we are given an enumeration $b$ of a nonrecursive, r.e. set $B$ and let $B^{t}=\left\{b_{i}: i<t\right\}$. We say that an attack is permitted at stage $t$ if each of its followers is $\geqslant b_{t}$. We say that a condition needs an initial attack at stage $t$ if either it has no attacks or each of 
its attacks needs attention but is not permitted. We devote stage $t$ to the highest priority condition without a three stage attack and either needing an initial attack or with a permitted attack needing attention. In the latter case we choose from the permitted attacks needing attention the earliest among those of greatest length (as sequences) (i.e. we give preference to extending two stage attacks over single stage attacks). We designate as invalid all prior stages devoted to lower priority conditions or devoted to later attacks on this condition. (Invalidating later attacks is necessary because followers of earlier attacks precede followers of later attacks whence attention to earlier attacks interferes with later attacks.)

Now the argument that a winning attack ensures its condition is as in the basic construction. It remains to show that each condition receives a winning attack. Suppose otherwise and let the $2 e$ th be the highest priority condition without a winning attack. (The case for an odd numbered condition is similar.) Further suppose that no stage after stage $t_{0}$ is devoted to a higher priority condition. Then no two stage attack on the $2 e$ th condition will receive attention after stage $t_{0}$ else the condition would have a winning attack. Since the $2 e$ th condition has no winning attack it will, by reductio ad absurdum, have infinitely many attacks which are never invalidated. Since as in the basic construction the need for attention persists each of these infinitely many attacks will need attention at all sufficiently late stages. Now the appearance at a stage $t>t_{0}$ of a two stage attack needing attention and with least follower $n$ signals that $B[n]=B^{t}[n]$ else some two stage attack will receive attention after stage $t_{0}$. Hence only finitely many of the attacks which persist can be two stage attacks (else $B$ would be recursive). Suppose $t_{1}>t_{0}$ is a stage after which no single stage attack receives attention. Then the appearance at a stage $t>t_{1}$ of a single stage attack needing attention and with least follower $n$ signals that $B[n]=B^{t}[n]$. Since there must be infinitely many such stages $B$ is recursive, contrary to hypothesis. Hence the $2 e$ th condition must have a winning attack.

Although we know from Ladner [7] that any attempt to improve the degree restriction in Theorem 4 must fail it is perhaps worthwhile examining why trapping fails. We would like to use the presence of trapped attacks needing attention to signal the final value of coding markers $c(n, t)$. But we cannot tell whether the presence at stage $t$ of a single stage attack needing attention in the $n$th trap indicates that this value is $c(n+1, t)$ or whether such an attack will be later extended to a two stage attack. Since there may be infinitely many persistent single stage attacks and since we cannot decide in which traps they are caught we cannot use the presence of two stage attacks (because they may later be invalidated by a single stage attack in a lower trap) to signal the the final value of a coding marker. 
3. Shared deficiency sets. We will show in this section that pairs of w-incomparable r.e. sets may share deficiency sets while pairs of 1-equivalent r.e. sets might not. $\mathbf{O}^{\prime}$ contains both sorts of pairs and below any r.e. degree $>\mathbf{0}$ there is an r.e. degree $>\mathbf{0}$ containing such a w-incomparable pair and an r.e. degree $>\mathbf{0}$ containing such a 1-equivalent pair. We also show that each r.e. degree $>\mathbf{0}$ contains a pair of tt-incomparable r.e. sets "enumerable in the same order" and hence sharing a deficiency set. Again from Ladner [7] we know the degree restrictions for w-reducibility are best possible; however in the case of 1-equivalence a better result may be possible.

R.e. sets $A$ and $A^{\prime}$ are defined by Rice [12] to be enumerable in the same order if they have enumerations $a$ and $a^{\prime}$ respectively such that $(\forall i, j)$ $\left[a_{i}<a_{j} \Leftrightarrow a_{i}^{\prime}<a_{j}^{\prime}\right]$. For such $a$ and $a^{\prime} H_{a}=H_{a^{\prime}}$ whence $A$ and $A^{\prime}$ share a deficiency set. Rice points out that sets enumerable in the same order are w-equivalent since, with $a$ and $a^{\prime}$ as above, given $n, i, j$ with $a_{i}<n<a_{j}$ deciding whether $n$ is in $A$ requires only knowledge of $A^{\prime} \cap\left\{m: a_{i}^{\prime}<m<a_{j}^{\prime}\right\}$. Hence the w-reducibility result is also best possible from this standpoint.

Theorem 5. Every r.e. degree $>0$ contains a pair of tt-incomparable r.e. sets enumerable in the same order.

Proof. The basic construction gives enumerations $a$ and $a^{\prime}$ in the same order of tt-incomparable r.e. sets $A$ and $A^{\prime}$ respectively. We take as our $2 e$ th $\left((2 e+1)\right.$ st) condition that $\varphi_{e}$ not be a tt-reduction of $A$ to $A^{\prime}\left(A^{\prime}\right.$ to $\left.A\right)$. Corresponding values of $a$ and $a^{\prime}$ will be defined at the same stage, at most one value per stage, and as before $j(t)$ will be the least $i$ with $a_{i}$ (and hence $\left.a_{i}^{\prime}\right)$ undefined prior to stage $t . A^{t}$ and $\left(A^{\prime}\right)^{t}$ denote $\left\{a_{i}: i<j(t)\right\}$ and $\left\{a_{i}^{\prime}: i<j(t)\right\}$ respectively. We describe attacks on even numbered conditions only, the case for odd numbered conditions being symmetric.

If stage $t$ is an initial attack on the $2 e$ th condition we designate $m(t)+1$ and $m(t)+2$ as followers of this attack and let $m(t+1)=m(t)+2$. We say that this attack needs attention at stage $t^{\prime}>t$ if it has not received attention at any intermediate stage and if $\varphi_{e}^{t^{\prime}}(m(t)+1)$ converges. In giving such attention we let $a_{j\left(t^{\prime}\right)}=m(t)+1=a_{j\left(t^{\prime}\right)}^{\prime}$ if $\varphi_{e}(m(t)+1)$ succeeds for $N-$ $\left(\left(A^{\prime}\right)^{t^{\prime}} \cup\{m(t)+1\}\right)$ and we let $a_{j\left(t^{\prime}\right)}=m(t)+2$ and $a_{j\left(t^{\prime}\right)}=m(t)+1$ otherwise. In either case we let

$$
m\left(t^{\prime}+1\right)=\operatorname{Max}\left(\left\{m\left(t^{\prime}\right)\right\} \cup \cup D_{\varphi_{e}(m(t)+1)}\right) .
$$

We have kept $a$ and $a^{\prime}$ in the same order by ensuring for each $i$ that $a_{i}$ and $a_{i}^{\prime}$ are chosen from some pair $m(t)+1$ and $m(t)+2$ and that no other values of $a$ or $a^{\prime}$ are ever chosen from this pair.

Now as before a single stage winning attack on the $2 e$ th condition indicates 
a divergence of $\varphi_{e}$. A two stage winning attack on the $2 e$ th condition, say containing stages $t<t^{\prime}$, ensures that $\varphi_{e}(m(t)+1)$ succeeds for $\bar{A}^{\prime}$ if and only if $m(t)+1 \in A$.

Trapping may be applied to the construction of $A$ as in previous cases. The conditions of the theorem, namely that $H_{a}=H_{a^{\prime}}$, ensure that $A$ and $A^{\prime}$ have the same degree.

THEOREM 6. Below every r.e. degree $>0$ there is an r.e. degree $>0$ containing a pair of w-incomparable r.e. sets sharing a deficiency set. Furthermore, $\mathbf{O}^{\prime}$ contains such a pair.

Proof. We again produce enumerations $a$ and $a^{\prime}$ of r.e. sets $A$ and $A^{\prime}$ respectively but now we only ensure that $H_{a}=H_{a^{\prime}}$. Again values of $a$ and $a^{\prime}$ will be defined simultaneously; $j(t)$ will be the least $i$ with $a_{i}$ undefined prior to stage $t$, and $A^{t}$ and $\left(A^{\prime}\right)^{t}$ will be $\left\{a_{i}: i<j(t)\right\}$ and $\left\{a_{i}^{\prime}: i<j(t)\right\}$ respectively. We ensure that $H_{a}=H_{a^{\prime}}$ by keeping $H_{a}^{j(t)}=H_{a}^{j(t)}$ at each stage $t$. (Recall that $H_{a}^{k}=\left\{i:(\exists j)\left[i<j<k \& a_{j}<a_{i}\right]\right\}$.) Again we describe attacks on even numbered conditions only.

At a stage $t$ of initial attack on the 2eth condition we designate $m(t)+1$ and $m(t)+2$ as followers of this attack and let $m(t+1)=m(t)+2$. We say that this attack needs attention at stage $t^{\prime}>t$ if it has not received attention at any intermediate stage and if $\varphi_{e}^{t^{\prime}}(m(t)+2)$ converges. In giving such attention we let $a_{j\left(t^{\prime}\right)}=m(t)+1=a_{j\left(t^{\prime}\right)}^{\prime}$; we let

$$
m\left(t^{\prime}+1\right)=\operatorname{Max}\left(\left\{m\left(t^{\prime}\right)\right\} \cup \cup D_{\varphi_{e}(m(t)+2)}\right)+1
$$

and we designate $m\left(t^{\prime}+1\right)$ as a new follower of this attack. Note that since stage $t$ is valid at stage $t^{\prime}$ one effect of stage $t^{\prime}$ is to put all numbers $i$ with $j(t) \leqslant i<j\left(t^{\prime}\right)$ into $H_{a}$ and $H_{a^{\prime}}$ (because for all such $i, m(t+1)<a_{i}$ ). We say that a two stage attack on the 2eth condition containing stages $t<t^{\prime}$ needs attention at stage $t^{\prime \prime}>t^{\prime}$ if it has not received attention at any intermediate stage and if $\varphi_{e}(m(t)+2)$ succeeds for $N-\left(\left(A^{\prime}\right)^{t^{\prime \prime}}\right)$ at stage $t^{\prime \prime}$. Note that by definition of $m\left(t^{\prime}+1\right)$ such success persists as long as stage $t^{\prime}$ remains valid. In giving such attention we define $a_{j\left(t^{\prime \prime}\right)}=m(t)+2$ and $a_{j\left(t^{\prime \prime}\right)}^{\prime}=m\left(t^{\prime}\right)$. We let $m\left(t^{\prime \prime}+1\right)=m\left(t^{\prime \prime}\right)$. Note that since stage $t^{\prime}$ is valid at stage $t^{\prime \prime}$ one effect of stage $t^{\prime \prime}$ is to put all numbers $i$ with $j\left(t^{\prime}\right)<i<j\left(t^{\prime \prime}\right)$ into $H_{a}$ and $H_{a^{\prime}}$ while keeping $j\left(t^{\prime}\right)$ out of $H_{a}$ and $H_{a^{\prime}}$ (because for all such $i a_{j\left(t^{\prime}\right)}<$ $\left.m\left(t^{\prime}+1\right)<a_{i}\right)$.

Now in case of a single stage winning attack on the $2 e$ th condition we have a divergence of $\varphi_{e}$. In case of a two stage winning attack containing stages $t<t^{\prime}$ we have $m(t)+2 \notin A$ while $\varphi_{e}(m(t)+2)$ fails for $\bar{A}^{\prime}$. In case of a three stage winning attack containing stages $t<t^{\prime}<t^{\prime \prime}$ we have that $m(t)+2 \in A$ 
while $\varphi_{e}(m(t)+2)$ succeeds for $\bar{A}^{\prime}$. Success here is preserved by the definition of $m\left(t^{\prime}+1\right)$.

To see that $H_{a}=H_{a^{\prime}}$ observe first that as long as a single stage attack, say containing stage $t$, persists (i.e. remains valid but is not extended) its lower follower, $m(t)+1$, threatens to put the same numbers into $H_{a}$ and $H_{a^{\prime}}$ (specifically all $i \geqslant j(t)$ with $a_{i}$ defined). Next observe that as long as a two stage attack, say containing stages $t<t^{\prime}$, persists the follower $m(t)+2$ threatens to put the same numbers into $H_{a}$ as the follower $m\left(t^{\prime}+1\right)$ threatens to put into $H_{a^{\prime}}$. Then since a second stage involved putting $m(t)+1$ into $A$ and $A^{\prime}$ and a third stage involved putting $m(t)+2$ into $A$ and $m\left(t^{\prime}+1\right)$ into $A^{\prime}, H_{a}=H_{a^{\prime}}$. (In case of attacks on odd numbered conditions interchange $a$ and $a^{\prime}$ as well as $A$ and $A^{\prime}$.)

$A$ and $A^{\prime}$ are seen to be complete as in Theorem 4. Since $H_{a}=H_{a^{\prime}}$, $A$ and $A^{\prime}$ have the same degree. Permitting may be applied to $A$ as in Theorem 4 to hold the degree of $A$ and $A^{\prime}$ down.

ThEOREM 7. Below every r.e. degree $>\mathbf{0}$ there is an r.e. degree $>\mathbf{0}$ containing a pair of 1-equivalent r.e. sets not sharing any deficiency sets. Furthermore $\mathbf{O}^{\prime}$ contains such a pair.

Proof. We will construct an r.e. set $A$ and let

$$
A^{\prime}=\{3 n: 3 n+1 \in A\} \cup\{3 n+1: 3 n \in A\} \cup\{3 n+2: 3 n+2 \in A\}
$$

whence $A$ and $A^{\prime}$ are 1-equivalent. We take as our $\langle e, f\rangle$ th condition that if $\varphi_{e}$ is an enumeration of $A$ and $\varphi_{f}$ is an enumeration of $A^{\prime}$ then $H_{\varphi_{e}} \neq H_{\varphi_{f}}$. We employ a marking function $v(e, t)$ defined as the least $n$ with $\varphi_{e}^{t}(n)$ divergent or $\varphi_{e}^{t}(n)=\varphi_{e}^{t}(m)$ for some $m<n$ to indicate initial segments of 1-1 convergence.

At a stage $t$ of initial attack on the $\langle e, f\rangle$ th condition we designate $3 m(t), 3 m(t)+1$ and $3 m(t)+2$ as followers of this attack and let $m(t+1)=$ $3 m(t)+2$. We say that such an attack needs attention at stage $t^{\prime}>t$ if it has not received attention at any intermediate stage and if

$$
\left\{\varphi_{e}(i): i<v\left(e, t^{\prime}\right)\right\}[3 m(t)+3]=A^{t^{\prime}}[3 m(t)+3]
$$

and

$$
\left\{\varphi_{f}(i): i<v\left(f, t^{\prime}\right)\right\}[3 m(t)+3]=\left(A^{\prime}\right)^{t^{\prime}}[3 m(t)+3] .
$$

In giving such attention we put $3 m(t)$ into $A$ (and hence we put $3 m(t)+1$ into $A^{\prime}$ ). We say that a two stage attack containing stages $t<t^{\prime}$ needs attention at stage $t^{\prime \prime}>t^{\prime}$ if it has not recieved attention at any intermediate stage and if 


$$
\begin{aligned}
& \left\{\varphi_{e}(i): i<v\left(e, t^{\prime \prime}\right)\right\}[3 m(t)+3]=A^{t^{\prime \prime}}[3 m(t)+3], \\
& \left\{\varphi_{f}(i): i<v\left(f, t^{\prime \prime}\right)\right\}[3 m(t)+3]=\left(A^{\prime}\right)^{t^{\prime \prime}}[3 m(t)+3],
\end{aligned}
$$

and $\varphi_{f}(j)=3 m(t)+1$ for some (unique) $j<v\left(e, t^{\prime \prime}\right)$. In giving such attention we put $3 m(t)+2$ into $A$ if $j \in H_{\varphi_{e}}^{v\left(e, t^{\prime \prime}\right)}$ or $3 m(t)+2<\varphi_{e}(j)$ and we put $3 m(t)+1$ into $A$ otherwise.

Now in case of a single stage or two stage winning attack either $\varphi_{e}$ is not an enumeration of $A$ or $\varphi_{f}$ is not an enumeration of $A^{\prime}$. In case of a three stage winning attack, say containing stages $t<t^{\prime}<t^{\prime \prime}$, if $\varphi_{e}$ and $\varphi_{f}$ are enumerations of $A$ and $A^{\prime}$ respectively we have that the unique $j$ referred to at stage $t^{\prime \prime}$ is in $H_{\varphi_{e}}$ if and only if $j \notin H_{\varphi_{f}}$

Completeness and permitting apply as in Theorem 4. In applying the completeness criterion choose $\left\langle e_{k}, f_{k}\right\rangle$ such that $\varphi_{e_{k}}$ and $\varphi_{f_{k}}$ are everywhere divergent or are enumerations of $A$ and $A^{\prime}$ respectively according as $k \notin W_{k}$ or $k \in W_{k}$.

4. Self-deficient sets and m-reducibility. The main result in this section is that below every r.e. degree $>\mathbf{0}$ there is an r.e. degree $>\mathbf{0}$ containing an r.e. set m-equivalent to each of its deficiency sets. The proof is facilitated by the introduction of self-deficient sets, r.e. sets $A$ with enumerations $a$ such that $A=H_{a}$, since by Jockusch [5, Theorem 3.2, 4.2(ii)] any deficiency set of a deficiency set $A$ is m-reducible to $A$. It is therefore sufficient to construct self-deficient sets m-reducible to all of their deficiency sets. m-equivalence is the best to be hoped for in this context since any r.e. set has deficiency sets whose symmetric difference is a singleton and simple sets whose symmetric difference is a singleton cannot be 1 -equivalent. $\left({ }^{3}\right)$

In the context of self-deficient sets we begin by showing that every r.e. degree contains one and we close by showing that some deficiency sets are not self-deficient. In the self-deficient set construction we use coding markers $c(n, t)$ as in trapping but we do not distinguish individual conditions in ensuring self-deficiency hence the construction has a different flavor than the preceding constructions. In the construction of the main theorem we combine the self-deficient set construction with $e$-state movement of markers to ensure nonrecursiveness and we apply permitting to push down the degree of the set constructed. $\left({ }^{4}\right)$ In the construction of a non-self-deficient, deficiency set we return to a basic construction and apply the completeness criterion to show that there is a complete, non-self-deficient, deficiency set. In this construction there

(3) I am indebted to C. G. Jockusch, Jr. for this observation.

( ${ }^{4}$ I am indebted to R. E. Ladner for observing that permitting is applicable here. 
is no a priori bound on the length of attack needed to satisfy a given condition, hence permitting cannot be applied while ensuring that each condition has a winning attack. Nonetheless a degree restricted result may be possible.

\section{THEOREM 8. Every r.e. degree contains a self-deficient set.}

Proof. It is easily verified that each co-infinite, recursive set is a deficiency set for each infinite, recursive set. However the combinatorial device needed to construct self-deficient sets of arbitrary degree is best illustrated by constructing an enumeration $a$ of a recursive set $A$ with $H_{a}=A$. In all constructions $j(t)$ is the least $i$ with $a_{i}$ undefined prior to stage $t, A^{t}=\left\{a_{i}: i<j(t)\right\}, m(0)=0$ and $m(t)=1+\operatorname{Max}\left(A^{t}\right)$ for $t>0$. We will have $A^{t}=H_{a}^{j(t)} \cup\{i: j(t) \leqslant i<$ $m(t)\}$ at each stage $t$ of each construction. (Recall that $H_{a}^{k}=\{i:(\exists j)[i<j<$ $\left.\left.k \& a_{j}<a_{i}\right]\right\}$.)

At stage $t$ in the construction of a recursive, self-deficient set we add the $(m(t)-j(t))+1$ numbers $m(t)+1$ through $m(t)+[(m(t)-j(t))+1]$ to $A$. We thus have that

$$
j(t+1)=j(t)+[(m(t)-j(t))+1]=m(t)+1
$$

and that

$$
m(t+1)=m(t)+[(m(t)-j(t))+1]+1 \text {. }
$$

We add these numbers to $A$ by defining $a_{j(t)+k}=m(t)+2+k$ for $k<$ $m(t)-j(t)$ (thus adding $m(t)+2$ through $m(t)+[(m(t)-j(t))+1])$ and defining $a_{m(t)}=m(t)+1$. This forces $\{i: j(t) \leqslant i<m(t)\} \subseteq H_{a}^{j(t+1)}$ and ensures that $A^{t+1}=H_{a}^{j(t+1)} \cup\{i: j(t+1) \leqslant i<m(t+1)\}$. It follows that $\bigcup_{t} A^{t}=\bigcup_{t} H_{a}^{j(t)}$ whence $A=H_{a}$.

Now let $b$ be an enumeration of an r.e. set $B$. As in trapping we use coding markers $c(n, t)$ to code $B$ in $A$ and restrict changes in $A$ modulo $b$. It follows as in trapping that $\mathbf{A}=\mathbf{B}$. In case $c\left(b_{t}, t\right)$ is not defined we proceed exactly as above and define $c(n, t+1)=m(t)$ for the least $n$ with $c(n, t)$ undefined. $\left(c(n, t+1) \cong c(n, t)\right.$ for all other $n$.) If $c\left(b_{t}, t\right)$ is defined we put all $k \notin A^{t}$ with $c\left(b_{t}, t\right) \leqslant k<m(t)$ into $A$. If there are $r$ such numbers we enumerate them in ascending order as $a_{m(t)}$ through $a_{m(t)+(r-1)}$. Next we define $c(n, t+1)=c(n, t)$ for $n \leqslant b_{t}$ and leave $c(n, t+1)$ undefined for $n>b_{t}$. Finally in analogy with the preceding case we add the $(m(t)-$ $j(t))+1$ numbers $m(t)+r+1$ through $m(t)+r+[(m(t)-j(t))+1]$ to $A$ by defining $a_{j(t)+k}=m(t)+r+2+k$ for $k<m(t)-j(t)$ (thus adding $m(t)+r+2$ through $m(t)+r+[(m(t)-j(t))+1])$ and defining $a_{m(t)+r}=$ $m(t)+r+1$. It follows that $j(t+1)=m(t)+r+1$ and $m(t+1)=m(t)+$ 
$r+[(m(t)-j(t))+1]+1$. We thus have that

$$
\{i: j(t+1) \leqslant i<m(t+1)\} \subseteq A^{t+1} \text { and }\{i: j(t) \leqslant i<m(t)\} \subseteq H_{a}^{j(t+1)} .
$$

Hence in order to show $A^{t+1}=H_{a}^{j(t+1)} \cup\{i: j(t+1) \leqslant i<m(t+1)\}$ it suffices to show $A^{t+1}[j(t)]=H_{a}^{j(t+1)}[j(t)]$. We have assumed that $A^{t}=H_{a}^{j(t)} \cup$ $\{i: j(t) \leqslant i<m(t)\}$ hence that $A^{t}[j(t)]=H_{a}^{j(t)}[j(t)]$. By construction there is a unique $t^{\prime}<t-1$ with $c\left(b_{t}, t\right)=m\left(t^{\prime}\right)$. If $k<j(t)$ belongs to $H_{a}^{j(t+1)}-$ $H_{a}^{j(t)}$ then $m\left(t^{\prime}\right)<\dot{a_{k}}$ whence $j\left(t^{\prime}\right) \leqslant k$ and $m\left(t^{\prime}\right) \leqslant k$ since $\left\{i: j\left(t^{\prime}\right) \leqslant i<\right.$ $\left.m\left(t^{\prime}\right)\right\} \subseteq H_{a}^{j\left(t^{\prime}+1\right)} \subseteq H_{a}^{j(t)}$. It follows by construction that $k \in A^{t+1}$. If $k<$ $j(t)$ belongs to $A^{t+1}-A^{t}$ then $m\left(t^{\prime}\right) \leqslant k$. Hence by construction $m\left(t^{\prime}\right)<$ $a_{k}$ since $c\left(b_{t}, t^{\prime \prime}\right)=m\left(t^{\prime}\right)$ for all $t^{\prime \prime}$ with $t^{\prime}<t^{\prime \prime} \leqslant t$. Then since $a_{m(t)}=$ $m\left(t^{\prime}\right)$ and $k<j(t)<m(t)$ we have $k \in H_{a}^{j(t+1)}$. It follows that $\bigcup_{t}^{m} A^{t}=$ $\bigcup_{t} H_{a}^{j(t)}$ and $A=H_{a}$.

It should be observed that if $B$ in Theorem 8 is high then $A$ is densesimple. Suppose $C=\left\{c_{0}<c_{1}<\cdots\right\}$ is an infinite recursive set. For each $n$ we can find a stage $t_{n}$ and an index $j_{n}$ such that $c\left(n, t_{n}\right)$ is defined, $c_{j_{n}}<$ $m\left(t_{n}\right)$, and $\left|N-\left(A^{t_{n}}\right)\left[c\left(n, t_{n}\right)\right]\right|<j_{n}$. Since $B$ is high $B[n+1] \neq$ $\left\{b_{t}: t<t_{n}\right\}[n+1]$ for almost all $n$. Hence by construction $\left|A\left[m\left(t_{n}\right)\right]\right|<j_{n}$ for almost all $n$. Since $c_{j_{n}}<m\left(t_{n}\right) \bar{A}$ majorizes $\left\{c_{j_{0}}<c_{j_{1}}<\cdots\right\}$ which in turn majorizes $C$. It follows that $A$ is dense-simple and from Robinson [13] that every high degree (i.e. degree with jump $0^{\prime \prime}$ ) contains a dense-simple, selfdeficient set.

THEOREM 9. Below every r.e. degree $>\mathbf{0}$ there is an r.e. degree $>\mathbf{0}$ containing an r.e. set m-equivalent to each of its deficiency sets.

PRoof. Given an enumeration $b$ of a nonrecursive, r.e. set $B$ we produce a self-deficient enumeration $a$ of a nonrecursive, r.e. set $A \leqslant_{T} B$. The use of coding markers $c(n, t)$ will differ from Theorem 8 in that $c\left(b_{t}, t\right)$ may not be put into $A$ even though defined and in that $c(n, t+1)$ may become undefined even though $c(n, t)$ is defined and no $c(m, t)$ for $m \leqslant n$ is put into $A$. If $c(n, t)$ is defined but $c(n, t+1)$ is not we say that $c(n, t)$ has been cancelled. If $c(n, t)$ is defined and not cancelled then $c(n, t+1)=$ $c(n, t)$.

As in Theorem 8 if any numbers below $m(t)$ are put into $A$ at stage $t$ then they are exactly the $k \in N-\left(A^{t}\right)$ with $c\left(b_{t}, t\right) \leqslant k<m(t)$ and the method of enumerating $A$ is exactly as in Theorem 8. Hence $A$ is self-deficient and by Jockusch [5] each deficiency set of $A$ is m-reducible to $A$. To see this in the context of this construction suppose $a^{\prime}$ is an enumeration of $A$. To decide whether a given $j$ is in $H_{a^{\prime}}$ find a stage $t>j$ with $a_{j}^{\prime}<m(t)$ and $\left\{a_{i}^{\prime}: i<t\right\}\left[a_{j}^{\prime}\right]=A^{t}\left[a_{j}^{\prime}\right]$. If $j \notin H_{a^{\prime}}^{t}$ then $j \in H_{a^{\prime}}$ if and only if 
$\operatorname{Max}\left(N-\left(A^{t}\right)\left[a_{j}^{\prime}\right]\right) \in A$ because any $k<a_{j}^{\prime}$ put into $A$ at stage $t^{\prime} \geqslant t$ is caused to be put into $A$ by $c\left(b_{t^{\prime}}, t^{\prime}\right) \leqslant k$ being put into $A$ whence, by construction all $k$ with $c\left(b_{t^{\prime}}, t^{\prime}\right) \leqslant k<m\left(t^{\prime}\right)$ (and in particular $\operatorname{Max}\left(N-\left(A^{t}\right)\left[a_{j}^{\prime}\right]\right)$ ) are put into $A$.

In addition to self-deficiency we have two types of conditions to satisfy. Our eth simplicity condition is that $A \cap W_{e} \neq \varnothing$ if $W_{e}$ is infinite. (Of course, $A$, being a deficiency set, is simple if nonrecursive but ensuring simplicity directly is combinatorially easier.) Our eth reduction condition is that $A \leqslant_{\mathrm{m}}$ $H_{\varphi_{e}}$ if $\varphi_{e}$ is an enumeration of $A$.

The construction employs three special marking functions. We define $n(0, t)=0$ and $n(e+1, t)=n(e, t)+\left|W_{e}^{t^{\prime}}\right|+1$ for each $t$ where $t^{\prime}$ is maximally $\leqslant t$ such that $A^{t^{\prime}} \cap W_{e}^{t^{\prime}}=\varnothing$. It is easy to see that $n(e, t)$ is increasing in $e$ and nondecreasing in $t$. We define $e(n, t)$ to be the largest $e$ with $n(e, t) \leqslant n$. It is also easy to see that $e(n, t)$ is nondecreasing in $n$ and nonincreasing in $t$. We use $e(n, t)$ and hence $n(e, t)$ in attending to the simplicity conditions while keeping $A \leqslant_{T} B$. Specifically we view $c(n, t)$, if defined, as assigned to the $e(n, t)$ th simplicity condition. Finally, as in Theorem 7 we define $v(e, t)$ to be the least $n$ with $\varphi_{e}^{t}(n)$ undefined or with $\varphi_{e}^{t}(n)=$ $\varphi_{e}^{t}(m)$ for some $m<n$. We use $v(e, t)$ to measure initial segments of 1-1 convergence in attending to the reduction conditions.

We will observe at the end of the construction that each of the coding markers $c(n, t)$ reaches a limit $c_{n} \notin A$. Since $e(n, t)$ is nonincreasing in $t$ each of these reaches a limit $e_{n}$. Our ultimate goal in satisfying the $e$ th reduction condition is to ensure that if $\varphi_{e}$ is an enumeration of $A$ then between any pair $c_{n}$ and $c_{n+1}$ with $e \leqslant e_{n}$ there is a $\varphi_{e}(j)$ with $j \notin H_{\varphi_{e}}$. If at stage $t$ we have $j<v(e, t)$ and $c(n, t)<\varphi_{e}(j)<c(n+1, t)$ with $A^{t}\left[\varphi_{e}(j)+1\right]=\left\{\varphi_{e}(i): i<v(e, t)\right\}\left[\varphi_{e}(j)+1\right]$ and $j \notin H_{\varphi_{e}}^{v(e, t)}$ then we know that as long as $\varphi_{e}$ enumerates $A$ any number $k$ with $c(n, t) \leqslant k<\varphi_{e}(j)$ to enter $A$ at stage $t$ or later will have to be enumerated as $\varphi_{e}\left(j^{\prime}\right)$ for some $j^{\prime} \geqslant v(e, t)>j$ and conversely any $\varphi_{e}\left(j^{\prime}\right)<c(n+1, t)$ with $j^{\prime} \geqslant v(e, t)$ will have to be forced into $A$ by some $c(m, t)$ for $m \leqslant n$. (The latter because the values $c\left(m, t^{\prime}\right)<c(n+1, t)$ with $t^{\prime} \geqslant t$ are among the values $c(m, t)$ for $m \leqslant n$.)

We divide each construction stage into two steps, the first devoted to reduction conditions and the second devoted to simplicity conditions. We begin the first step by searching the defined coding markers for a pair $c(n, t)$, $c(n+1, t)$ such that for some $e \leqslant e(n, t)$ there is a $j<v(e, t)$ with $j \notin$ $H_{\varphi_{e}}^{v(e, t)}, c(n, t)<\varphi_{e}(j)$ and $A^{t}\left[\varphi_{e}(j)+1\right]=\left\{\varphi_{e}(i): i<v(e, t)\right\}\left[\varphi_{e}(j)+1\right]$ but such that for any such $j, c(n+1, t)<\varphi_{e}(j)$. If such a pair exists we pick the least such pair (i.e. the least $n$ with $c(n, t), c(n+1, t)$ such a pair) and cancel 
all $c(n, t)$ for $m \geqslant n+1$. We begin the second step by determining whether $c\left(b_{t}, t\right)$ is defined and not cancelled in the first step. If so we then determine whether $A^{t} \cap W_{e\left(b_{t}, t\right)}^{t}=\varnothing$ while $\left\{k: c\left(b_{t}, t\right) \leqslant k<m(t)\right\} \cap W_{e\left(b_{t}, t\right)}^{t} \neq \varnothing$. If this is also the case we decide to put all $k \notin A^{t}$ with $c\left(b_{t}, t\right) \leqslant k<m(t)$ into $A$ and otherwise we decide to put no numbers below $m(t)$ into $A$. In the former case we cancel all $c(m, t)$ for $m \geqslant b_{t}$. (We cancel $c\left(b_{t}, t\right)$ here contrary to Theorem 8 in order to have $c_{b_{t}} \notin A$.) In the latter case we define $c(n, t+1)=m(t+1)$ for the least $n$ with $c(n, t)$ undefined or cancelled in the first step.

We observe first that the limit $c_{n}$ exists for each $n$. Clearly $c_{0}=0$ unless at some stage $t, b_{t}=0$ and second step action causes $c(0, t)$ to be cancelled. In the latter case no numbers below $m(t+1)$ will enter $A$ at stage $t+1$ whence $c(0, t+2)=m(t+1)$ will be defined (as in Theorem 8) and since $b$ is $1-1$ and no first step action can cancel $c\left(0, t^{\prime}\right)$ at any stage $t^{\prime}$, we have $c_{0}=c(0, t+2)$. Suppose $c_{n}$ exists and let $t_{0}$ be a stage by which $c_{n}$ and $e_{n}$ are reached and such that $n+1<b_{t}$ for all $t \geqslant t_{0}$. Then if $t \geqslant t_{0}$ and $c(n+1, t)$ is defined and cancelled this cancellation will be caused by first step action relative to the pair $c(n, t)$, $c(n+1, t)$. If $e$ and $j$ are as in the first step then the first step will never apply relative to $e$ and the pair $c\left(n, t^{\prime}\right), c\left(n+1, t^{\prime}\right)$ at a stage $t^{\prime}>t$ unless $j \in H_{\varphi_{e}}^{v\left(e, t^{\prime}\right)}$. But in this case $\varphi_{e}\left(j^{\prime}\right)<\varphi_{e}(j)$ for some $j^{\prime} \geqslant v(e, t)$ while, on the other hand, $A^{t}\left[\varphi_{e}(j)+1\right]=A^{t^{\prime}}\left[\varphi_{e}(j)+1\right]$ since $c(n, t)=c_{n}$ and $c\left(n+1, t^{\prime}\right) \geqslant m(t)>\varphi_{e}(j)$. Hence the first step will never apply relative to $e$ and the pair $c\left(n, t^{\prime}\right), c\left(n+1, t^{\prime}\right)$ at any stage $t^{\prime}>t$. Since for $t \geqslant t_{0}$ we will define $c(n+1, t+1)=m(t+1)$ whenever $c(n+1, t)$ is not defined we have by reductio ad absurdum that $c_{n+1}$. exists and by induction that each limit exists.

To see that $A$ is simple observe first that $A$ is co-infinite since each $c_{n} \in \bar{A}$. Suppose for purposes of contradiction that $A$ is not simple and let $f$ be minimal with $W_{f}$ an infinite subset of $\bar{A}$. By the minimality of $f$ only finitely many $e_{n}$ are $<f$. Let $n_{0}$ be minimal with $e_{n_{0}}=f$. By hypothesis $e_{n}=f$ for all $n \geqslant n_{0}$. If $\varphi_{e}$ is not an enumeration of $A$ then only finitely many first steps will apply relative to $e$ since either $v(e, t)$ will reach a limit or there will be a largest $j$ with $A^{t}\left[\varphi_{e}(j)+1\right]=\left\{\varphi_{e}(i): i<v(e, t)\right\}\left[\varphi_{e}(j)+1\right]$ for some $t$. Let $t_{0}$ be a stage by which the limits $c_{n_{0}}$ and $e_{n_{0}}$ are reached and after which no first step applies relative to any $e \leqslant f$ for which $\varphi_{e}$ is not an enumeration of $A$. It is not difficult to verify by arguments similar to those showing that the limits $c_{n}$ exist that if the first step of a stage $t>t_{0}$ applies relative to $e \leqslant f$ and a pair $c(n, t), c(n+1, t)$ then no later stage $t^{\prime}>t$ applies to $e$ relative to the pair $c\left(n, t^{\prime}\right), c\left(n+1, t^{\prime}\right)$ if $c\left(n, t^{\prime}\right)=c(n, t)$. It is also not difficult to see that for each $e \leqslant f$ with $\varphi_{e}$ an enumeration of $A$ and each $n \geqslant n_{0}$ there will be a $j \notin H_{\varphi_{e}}$ with $c_{n}<\varphi_{e}(j)<$ $c_{n+1}$. Since $W_{f}$ is an infinite subset of $\bar{A}$ we know that for each $n$ there will be a stage $t$ with $A^{t} \cap W_{f}^{t}=\varnothing$ while $\{k: c(n, t) \leqslant k<m(t)\} \cap W_{f}^{t} \neq \varnothing$. Hence, by the 
second step, it suffices in order to conclude for $n \geqslant n_{0}$ that $B[n+1]=$ $\left\{b_{i}: i<t\right\}[n+1]$ to have $t>t_{0}, e(n, t)=f,\{k: c(n, t) \leqslant k<m(t)\} \cap w_{f}^{t} \neq \varnothing$ and to have for each $e \leqslant f$ with $\varphi_{e}$ an enumeration of $A$ a $j \notin H_{\varphi_{e}}^{(e, t)}$ with $c(n, t)<\varphi_{e}(j)$ $<c(n+1, t)$. This being contrary to $B$ 's nonrecursiveness we have the desired contradiction.

It is now easy to verify that $A \leqslant_{\mathrm{m}} H_{\varphi_{e}}$ if $\varphi_{e}$ is an enumeration of $A$. Given $e$ let $n_{0}$ be minimal with $e_{n_{0}}=e$ and let $t_{0}$ be a stage by which $c_{n_{0}}$ and $e_{n_{0}}$ are reached and after which no first step applies relative to an $e^{\prime}<e$ with $\varphi_{e^{\prime}}$ not an enumeration of $A$. Then, for $n \geqslant n_{0}, t>t_{0}$ and $j \notin H_{\varphi_{e}}^{v(e, t)}$ with $c(n, t)$ $<\varphi_{e}(j)<c(n+1, t)$ and $A^{t}\left[\varphi_{e}(j)+1\right]=\left\{\varphi_{e}(i): i<v(e, t)\right\}\left[\varphi_{e}(j)+1\right]$, we know that for any $k \notin A^{t}$ with $c(n, t) \leqslant k<\varphi_{e}(j), k \in A$ if and only if $j \in H_{\varphi_{e}}$. Hence $A \leqslant_{\mathrm{m}} H_{\varphi_{e}}$.

Finally, since $c(n, t)$ is increasing in $n$ (where defined) and nondecreasing in $t$ in the sense that $c(n, t) \leqslant c\left(n, t^{\prime}\right)$ wherever both are defined and $t \leqslant t^{\prime}$, we have $A \leqslant_{T} B$ (in fact $A \leqslant_{\mathrm{w}} B$ ). This because $A[c(n, t)]=A^{t}[c(n, t)]$ whereever $B[n]=\left\{b_{i}: i<t\right\}[n]$ and because $c_{n}$ exists for each $n$.

\section{TheOREM 10. There is a complete, non-self-deficient deficiency set.}

PROoF. We return to the methods of $\S 1-\S 3$, using a basic construction to produce an enumeration $a$ of an r.e. set $A$ which, by Soare's completeness criterion, is complete. $H_{a}$ will be non-self-deficient and hence, by opening remarks in Theorem 8, nonrecursive. Of course, by Soare's completeness criterion for $A$, $H_{a}$ is complete. We are unable to apply permitting, lacking an a priori bound on the length of attack for a given condition.

We take as our $e$ th condition that $\varphi_{e}$ not be an enumeration of both $H_{a}$ and $H_{\varphi_{e}}$. At each stage $t$ we define $a_{2 t}=M(t)+t+3$ and we define $a_{2 t+1}$ ensuring that $a_{2 t+1}<a_{2 t}$ so that $\{2 i: i \in N\} \subseteq H_{a}$. We let $m(t+1)=m(t)+$ $t+4$ and if stage $t$ is an initial attack we let $a_{2 t+1}=m(t)+t+2$ and designate all $k$ with $m(t) \leqslant k \leqslant m(t)+t+1$ as followers of this attack. If stage $t$ is not an initial attack but is devoted instead to extending an existing attack we let $a_{2 t+1}$ be the largest follower of this attack not in $A^{t}$ where $A^{t}=\left\{a_{i}: i<2 t\right\}$. Hence an attack begun at stage $t$ may have length $t+3$ and since we have no a priori knowledge of when the winning attack on the $e$ th condition is initiated we have no a priori bound on the length of this attack.

By basic construction rules any condition without an existing attack needs an initial attack and each stage is devoted to the highest priority (lowest numbered) condition in need. A single stage attack on the $e$ th condition containing stage $t_{0}$ needs attention at stage $t>t_{0}$ if $2 t_{0}+1 \notin\left\{\varphi_{e}(i): i<v(e, t)\right\} ; 2 t_{0}+1<v(e, t)$, and $\left\{2 i: i \leqslant t_{0}\right\} \subseteq\left\{\varphi_{e}(i): i<v(e, t)\right\}$. (Here as in Theorems 7 and $9 v(e, t)$ marks initial segments of 1-1 convergence.) A multiple stage attack on the $e$ th condition containing stages $t_{0}<\cdots<t_{r}$ needs attention at a stage $t>t_{r}$ if $\varphi_{e}(j) \in$ $\left\{2 i+1: i \leqslant t_{0}\right\}$ for some $j$ with $v(e, t-1) \leqslant j<v(e, t)$.

Notice that an attack begun at stage $t_{0}$ can have length at most $t_{0}+3$ since 
each stage beyond the second stage in this attack corresponds to the enumeration of a unique odd number between 1 and $2 t_{0}+1$. It follows that each $a_{2 t+1}$ is well defined and by basic construction rules, that each condition has a winning attack since no attack begun at stage $t_{0}$ and of length $t_{0}+3$ can need attention.

Now if the winning attack on the $e$ th condition is a single stage attack containing stage $t_{0}$ then either $\varphi_{e}$ is not $1-1,\{2 i: i \in n\} \nsubseteq \operatorname{Rng}\left(\varphi_{e}\right)$, or $2 t_{0}+1 \in$ $\operatorname{Rng}\left(\varphi_{e}\right)-H_{a}$. If the winning attack is a two stage attack containing stages $t_{0}<$ $t_{1}$ then $2 t_{0}+1 \in H_{a}-\operatorname{Rng}\left(\varphi_{e}\right)$ unless $\varphi_{e}$ is not an enumeration. If the winning attack contains stages $t_{0}<\cdots<t_{r}$ where $r>1$ then there is a largest $j$ satisfying $v\left(e, t_{r}-1\right) \leqslant j<v\left(e, t_{r}\right)$ with $\varphi_{e}(j) \in\left\{2 i+1: i \leqslant t_{0}\right\}$. Since $t_{r}$ is the final stage in this attack $j \notin H_{\varphi_{e}}$ or $\varphi_{e}$ is not an enumeration. By construction we have $a_{2 t_{r}+1}<a_{j}$ since $2 t_{0}+1<v\left(e, t_{1}\right) \leqslant v\left(e, t_{r}-1\right)<j$ and we have $j \leqslant t_{r}$ $<2 t_{r}+1$ since $j<v\left(e, t_{r}\right)$ and, by convention, $\varphi_{e}^{t}(n)$ is undefined for $n>t$. Hence $j \in H_{a}-H_{\varphi_{e}}$ or $\varphi_{e}$ is not an enumeration. In any case $\varphi_{e}$ is not an enumeration of both $H_{a}$ and $H_{\varphi_{e}}$.

To see that $A$ is complete observe that for each $k$ we may find an index $e_{k}$ such that $\varphi_{e_{k}}$ enumerates $H_{a}$ if $k \in W_{k}$ and is everywhere undefined otherwise.

\section{REFERENCES}

1. J. C. E. Dekker, Two notes on recursively enumerable sets, Proc. Amer. Math. Soc. 4 (1953), 495-501. MR 15, 385.

2. - A theorem on hypersimple sets, Proc. Amer. Math. Soc. 5 (1954), $791-$ 796. MR 16, 209.

3. J. C. E. Dekker and J. Myhill, Retraceable sets, Canad. J. Math. 10 (1958), 357-373. MR 20 \#5733.

4. R. M. Friedberg and H. R. Rogers, Reducibility and completeness for sets of integers, Z. Math. Logik Grundlagen Math. 5 (1959), 117-125. MR 22 \#3682.

5. C. G. Jockusch, Jr., Semirecursive sets and positive reducibility, Trans. Amer. Math. Soc. 131 (1968), 420-436. MR 36 \#3649.

6. R. E. Ladner, Mitotic recursively enumerable sets. J. Symbolic Logic 38 (1973), 199-211.

7. - A completely mitotic non-recursive r.e. degree, Trans. Amer. Math. Soc. 184 (1973), 479-507.

8. D. A. Martin, Classes of recursively enumerable sets and degrees of unsolvability, Z. Math. Logik Grundlagen Math. 12 (1966), 295-310. MR 37 \#68.

9. - A theorem on hyperhypersimple sets, J. Symbolic Logic 28 (1963), 273278. MR 31 \#2145.

10. J. Myhill, Creative sets, Z. Math. Logik Grundlagen Math. 1 (1955), 97-108. MR 17, 118.

11. E. L. Post, Recursively enumerable sets of positive integers and their decision problems, Bull. Amer. Math. Soc. 50 (1944), 284-316. MR 6, 29.

12. H. G. Rice, Recursive and recursively enumerable orders, Trans. Amer. Math. Soc. 83 (1956), 277-300. MR 18, 712.

13. R. W. Robinson, $A$ dichotomy of the recursively enumerable sets, $Z$. Math. Logik Grundlagen Math. 14 (1968), 339-356. MR 38 \#5623. 
14. G. E. Sacks, Degrees of unsolvability, Princeton Univ. Press, Princeton, N. J., 1963. MR 32 \#4013.

15. R. I. Soare, The Friedberg-Muchnik theorem re-examined, Canad. J. Math. 24 (1972), 1070-1078.

16. C. E. M. Yates, Recursively enumerable sets and retracing functions, $Z$. Math. Logik Grundlagen Math. 8 (1962), 331-345. MR 26 \#3598.

17. - Three theorems on the degrees of recursively enumerable sets, Duke Math. J. 32 (1965), 461-468. MR 31 \#4721.

DEPARTMENT OF MATHEMATICS, UNIVERSITY OF CALIFORNIA, IRVINE, CALIFORNIA 92664

Current address: 2983 Alexander Road, Laguna Beach, California 92651 